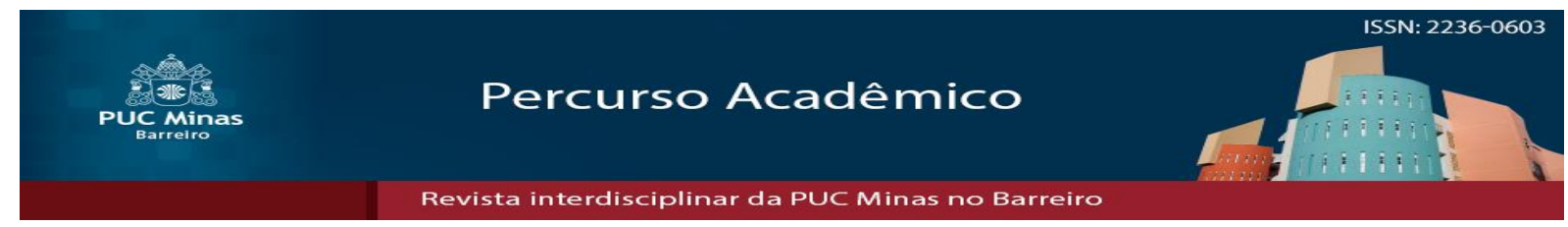

\title{
Experiência extensionista em Engenharia de Produção: desafios e oportunidades
}

Extensionist experience in Production Engineering: challenges and opportunities

\author{
Alessandra Lopes Carvalho ${ }^{1}$ \\ Luciano Andrade ${ }^{2}$ \\ Pedro Ivo Alexandre Oliveira ${ }^{3}$ \\ Ricardo Cobério Lery Santos ${ }^{4}$
}

\section{Resumo}

Este trabalho é fruto de projeto de extensão concebido com o intuito de contribuir para o desenvolvimento de microempresas pertencentes ao entorno da universidade na qual foi originado o estudo. Após uma etapa inicial de triagem e escolha das empresas com interesse em participar do projeto, foi escolhida uma empresa específica como projeto piloto. Posteriormente 0 projeto foi implementado em outras microempresas, caracterizando, portanto, um estudo de múltiplos casos. Foram utilizadas abordagens de pesquisa qualitativas e quantitativas sendo aplicados diferentes métodos de coleta e tratamento de dados como análise de documentos e entrevistas semiestruturadas. Após a etapa de diagnóstico dos principais problemas encontrados em cada empresa participante do projeto, foram utilizadas ferramentas da qualidade para a busca de possíveis soluções. Posteriormente foi elaborado um plano de ação específico para cada empresa. Os resultados obtidos demostram claras possibilidades de melhoria a partir de soluções simples e de baixo custo. Conclui-se ainda que a demanda das micro e

\footnotetext{
${ }^{1}$ Doutora em Engenharia Elétrica na área de Confiabilidade e Mestrado em Modelagem e Automação de Processos pela Universidade Federal de Minas Gerais (UFMG). Atualmente leciona Confiabilidade, Mantenabilidade e Disponibilidade de Sistemas na Pontifícia Universidade Católica de Minas Gerais (PUC MG). Brasil. E-mail: alessandralcarvalho@pucminas.br

${ }_{2}^{2}$ Mestre em Engenharia Metalúrgica e de Minas pela Universidade Federal de Minas Gerais (UFMG). Graduado em Engenharia Metalúrgica pela Universidade Federal de Minas Gerais (UFMG). Técnico metalurgista pelo Colégio São Francisco Xavier em Ipatinga Minas Gerais. Atua como professor assistente no Instituto Politécnico IPUC da Pontifícia Universidade Católica de Minas Gerais (PUC MG). Brasil. E-mail: lucianoandrade@pucminas.br

${ }^{3}$ Mestrado em Ciências da Computação pela Universidade Federal de Minas Gerais (PUC MG). Graduado em Ciência da Computação pela Universidade Federal de Ouro Preto (UFOP). Atualmente é professor Assistente IV - rti da Pontifícia Universidade Católica de Minas Gerais (PUC MG). Brasil. E-mail: ivo@pucminas.br

${ }^{4}$ Mestrado em Engenharia de Produção e Graduação em Engenharia Civil pela Universidade Federal de Minas Gerais (UFMG). Atualmente é professor assistente da Universidade Salgado de Oliveira e professor assistente - Nível 4 na Pontifícia Universidade Católica de Minas Gerais. Brasil. E-mail: rlery@pucminas.br
} 
pequenas empresas por suporte técnico vem de encontro à busca por parte das universidades pela efetivação do seu compromisso social, gerando benefícios para ambas as partes.

Palavras-chave: Extensão Universitária; Micro Empresa; Ferramentas da Qualidade.

\section{Abstract}

This work is the result of an extension project designed to contribute to the development of microenterprises belonging to the university environment in which the study originated. After an initial stage of screening and choice of companies interested in participating in the project, a specific company was chosen as a pilot project. Subsequently the project was implemented in other micro-enterprises, characterizing, therefore, a study of multiple cases. Qualitative and quantitative research approaches were used, and different methods of data collection and treatment were applied, such as document analysis and semi-structured interviews. After the diagnostic phase of the main problems found in each company participating in the project, quality tools were used to search for possible solutions. Subsequently, a specific action plan was drawn up for each company. The results show clear possibilities for improvement from simple and low cost solutions. It is also concluded that the demand of micro and small companies for technical support comes against the search by the universities for the realization of their social commitment, generating benefits for both parties.

Keywords: University Extension. Micro enterprise. Quality tools.

\section{Introdução}

Segundo o SEBRAE (2015), as microempresas representam aproximadamente $97 \%$ do total de empresas brasileiras. Essa tendência, que atinge o mundo como um todo, é consequência da facilidade do acesso a informações, que leva a uma abertura para o empreendedorismo.

Entretanto, ainda segundo o SEBRAE (2015), 27\% de todas as novas empresas fundadas no Brasil fecham as portas no primeiro ano de vida. Existem várias possíveis explicações para este fato: a empresa pode ter começado como uma empresa familiar e não conseguiu se estruturar, a 
competição com as grandes empresas ou até mesmo falta de conhecimentos e embasamento teórico em práticas de gestão.

No caso específico do Brasil, a falta de planejamento é uma característica cultural enraizada. Assim, muitas empresas são abertas devido a um pensamento equivocado de lucro e não se planejam para situações diversas que podem atingir o mercado. De maneira geral, os modelos de estratégia encontrados na literatura são predominantemente voltados para grandes empresas. Entretanto, pequenas e médias empresas necessitam de estratégias que respeitem suas peculiaridades (PIOVEZAN; BARBIN; CARVALHO, 2008).

É sabido que as microempresas geram desenvolvimento local. Assim, a criação de condições que fortaleçam e gerem mais emprego e renda são facilitadoras de um ciclo de prosperidade. Neste contexto, segundo a ABEPRO (2008), produzir é mais que simplesmente utilizar conhecimento científico e tecnológico. É necessário integrar questões de naturezas diversas, atentando para critérios de qualidade, eficiência, custos porem enfatizando fatores humanos e ambientais.

A Engenharia de Produção, ao voltar a sua ênfase para as dimensões do produto e do sistema produtivo, vincula-se fortemente com as ideias de projetar produtos, viabilizar produtos, projetar sistemas produtivos, viabilizar sistemas produtivos, planejar a produção, produzir e distribuir produtos que a sociedade valoriza. Essas atividades, tratadas em profundidade e de forma integrada, são fundamentais para a elevação da competitividade do país.

Segundo Larozinki Neto e Leite (2010), os problemas da área de Engenharia de Produção demandam abordagens que transitam entre a pluri, a inter e a transdisciplinaridade. A necessidade dessas abordagens é resultado da diversidade de variáveis que envolvem as áreas do conhecimento relacionadas aos problemas da Engenharia de Produção. Por exemplo, a pesquisa sobre o funcionamento de um processo produtivo necessita "observar" o processo do ponto de vista do seu arranjo físico, dos fluxos de materiais, dos fluxos de informação, das relações sociais que se estabelecem entre os envolvidos, do conhecimento e a capacidade de aprendizagem dos indivíduos, dos fatores ambientais que incidem na operação do processo, das condições de trabalho, das relações formais e informais, entre outros. É 
possível abordar apenas um desses aspectos, mas deve-se ter consciência de que essa abordagem será parcial e irá limitar a análise do problema.

Considerando a complexidade, a abrangência e as características pluri, inter e transdisciplinares inerentes à Engenharia de Produção, compete aos educadores lidarem constantemente com o desafios naturalmente impostos pelo processo ensino/aprendizagem (CARVALHO; NERY, 2015). Diante deste contexto é imperativa a busca de ferramentas que auxiliem a compreensão e a assimilação de conceitos por parte do corpo discente, através da articulação entre o ensino, a pesquisa e a extensão.

Sabe-se que a aprendizagem de qualquer conceito ocorre com maior efetividade se, associadas à transmissão de conceitos teóricos, for possível realizar uma aplicação prática que facilite o processo de ensino/aprendizagem. Uma forma de possibilitar a aplicação prática de conceitos teóricos é através de atividades extensionistas.

Segundo o Plano Nacional de Extensão Universitária, entende-se a extensão como um processo educativo, cultural e científico que articula o ensino e a pesquisa de forma indissociável e viabiliza a relação transformadora entre universidade e sociedade (FÓRUM NACIONAL DE PRÓ-REITORES DE EXTENSÃO DAS UNIVERSIDADES PÚBLICAS BRASILEIRAS, 2001). Considerando o contexto de várias universidades (CRUZ et al., 2010; RODRIGUES et al., 2013; ANJOS et al., 2015; CASTRO e tal. 2017; LAGE; DIAS, 2016; NEVES et al., 2017; SOUZA; ATAYDE, 2017), percebe-se a extensão como prática produtora e disseminadora de conhecimentos advindos da comunidade acadêmica. Entende-se ainda que a extensão atue como instrumento de busca a respostas para questões sociais possibilitando a formação de profissionais que sejam tecnicamente competentes e eticamente comprometidos.

Diante da problemática exposta, objetiva-se selecionar empresas de pequeno porte dispostas a se submeterem a um processo de interação e parceria empresa-universidade. Espera-se que as empresas selecionadas compartilhem seus problemas de forma a buscar soluções através da aplicação de ferramentas e práticas intrínsecas a Engenharia de Produção. Este trabalho apresenta os resultados referentes à primeira empresa estudada (projeto piloto) 
e outras duas empresas, caracterizando, portanto, um estudo de múltiplos casos.

\section{Engenharia de Produção}

Conforme exposto anteriormente, a Engenharia de Produção é essencialmente multidisciplinar. Segundo a ABEPRO (2008), a Engenharia de Produção pode ser subdividida em nove grandes áreas conforme apresentado na Figura 1. Maiores detalhes podem ser obtidos em Batalha (2008), Slack; Chambers; Johnston (2010), Venanzi; Silva (2016). O foco deste trabalho é a aplicação de ferramentas da qualidade, portanto concentra-se na área Engenharia da Qualidade.

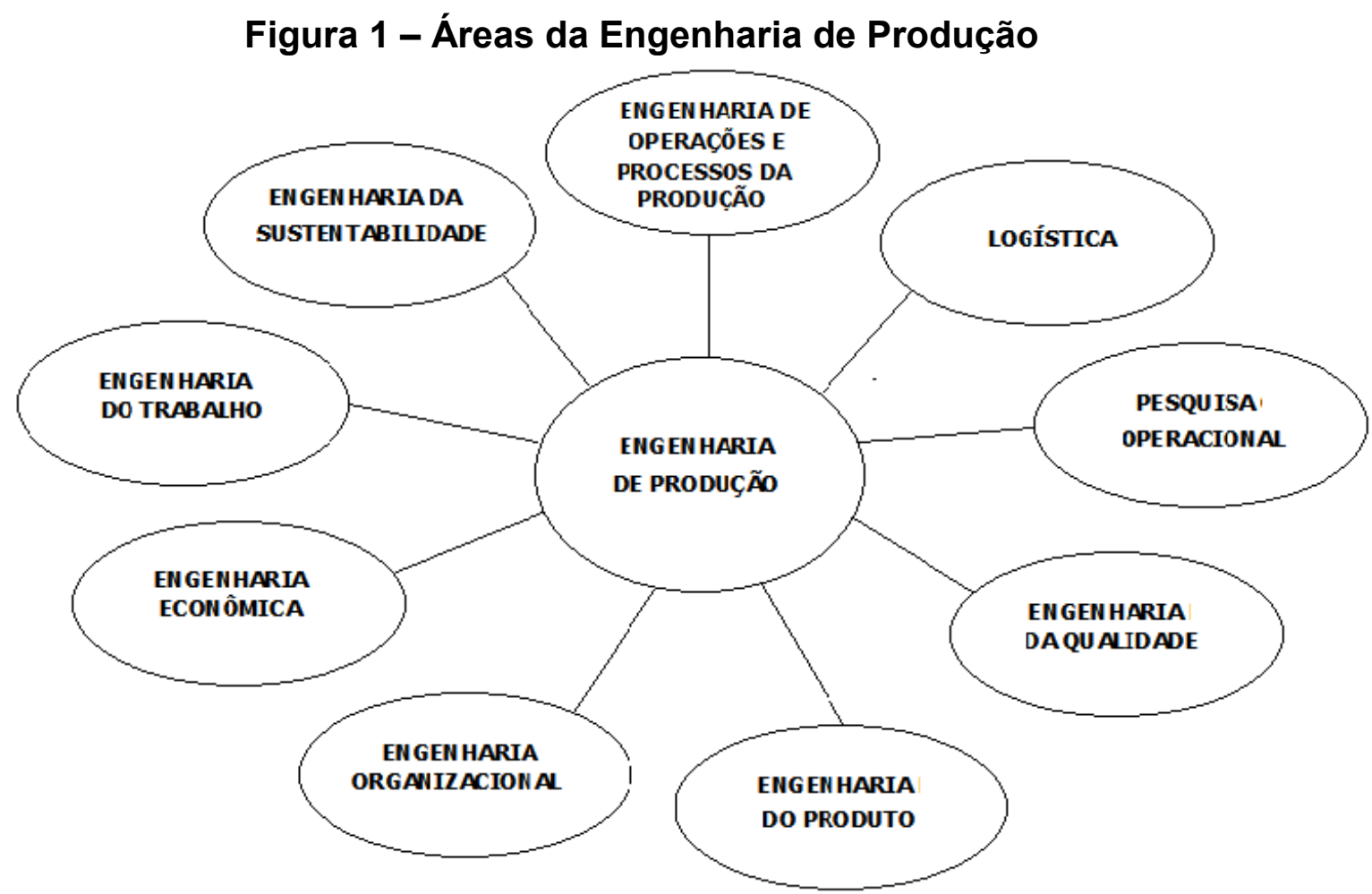

Fonte: Elaborada pelos autores

\subsection{Engenharia da qualidade}

A qualidade é algo inerente ao ser humano e o pode estar relacionada genericamente ao nível de satisfação oferecida por produto ou processo do ponto de vista do usuário, do produto em si, da produção ou do valor agregado. Esta satisfação pode ser desdobrada em termos de características específicas 
peculiares a cada situação como durabilidade, conformidade, atendimento (no caso de serviços), confiabilidade, dentre outros.

A importância da busca contínua pela qualidade tem assumido novas proporções nas últimas décadas, devido à concorrência crescente na indústria como um todo. Existe ainda uma constante preocupação com a diminuição de custos e prazos de entrega exigindo uma concepção integrada e dinâmica dos processos produtivos.

O termo qualidade tem sido estudado por vários pontos de vista diferentes podendo ser citados como exemplo a Filosofia, a Economia e o Marketing. Podem ser identificadas várias abordagens para a definição da qualidade (TOLEDO et al., 2013). A abordagem transcendente trata a qualidade como um conceito abstrato com característica de excelência inata e definição imprecisa. A qualidade baseada no usuário, do ponto de vista do marketing, pode ser traduzida como a capacidade de satisfazer desejos tendo uma forte característica, portanto, de adequação ao uso. Tomando por base 0 produto, a qualidade assume características precisas e mensuráveis refletindo diferenças na quantidade de um atributo ou ingrediente. A abordagem baseada na produção relaciona-se à conformidade com as especificações e finalmente a abordagem baseada no valor define a qualidade em termos de custos e preços (PALADINI, 2011).

O conceito de qualidade, como é conhecido em termos atuais, está intimamente relacionado à história do Japão após a Segunda Guerra (CARPINETTI, 2012). Inicialmente, o foco da Qualidade foi o produto (influenciado por fatores históricos e devido à necessidade de sobrevivência) tendo posteriormente evoluído para a metodologia TQC (Total Quality Control) direcionada ao gerenciamento.

Portanto, percebe-se historicamente que a qualidade deixou o chão de fábrica e com o passar do tempo assumiu uma posição de importância estratégica em qualquer organização.

Dentre as ferramentas clássicas de qualidade podem ser citadas Histograma, Folha de Controle, Gráfico de Pareto, Diagrama de Causa e Efeito, Diagrama de Concentração de Defeitos, Diagrama de Dispersão e Gráfico de Controle (MONTGOMERY, 2016). Outros programas e métodos para a melhoria da qualidade podem ser encontrados em Rodrigues (2014). 


\section{Materiais e métodos}

O desenvolvimento deste trabalho ocorreu por meio de pesquisas com características qualitativas e quantitativas utilizando como método o estudo de casos múltiplos (CAUCHICK MIGUEL, 2012). Foram realizadas coletas de dados primários e secundários utilizados diferentes métodos de coleta e tratamento de dados como análise de documentos e entrevistas semiestruturadas.

A execução deste projeto foi subdividida em três grandes fases. A primeira fase envolveu a escolha das empresas participantes do projeto e a compreensão do contexto. Posteriormente, em uma segunda fase escolheu-se uma empresa para implantação do projeto em escala piloto. Finalmente, após o aprendizado proporcionado pelo projeto piloto foi realizado um estudo de múltiplos casos. Apresenta-se a seguir o detalhamento de todas as fases.

Durante a primeira fase realizou-se um diagnóstico com objetivo de identificar as empresas que possivelmente poderiam se interessar em participar do projeto, utilizando-se principalmente o site da FIEMG (2016). Foi identificado um número três vezes superior a necessidade do projeto, utilizando-se como critério o porte da empresa (priorizando-se as menores empresas) e a proximidade física com a Universidade, de forma a facilitar a logística de deslocamento dos alunos participantes no projeto.

Foi escolhida como projeto piloto uma empresa de pequeno porte fabricante de cortinas. Considerando-se o método estudo de caso, foram realizadas visitas técnicas na empresa foco do estudo piloto a fim de diagnosticar possíveis falhas e sugerir melhorias no seu processo de produção. É importante ressaltar que a empresa tem uma produção bastante customizada com diferentes operações e lead time distintos na sua linha de produção. Essa peculiaridade deve-se ao fato da maioria dos produtos serem fabricados sob encomenda (customizados), ou seja, de acordo com exigências do cliente (CARVALHO et al., 2016).

Após a compreensão de todas as etapas do processo produtivo foram realizadas observações diretas focando na operação dos funcionários e as suas formas de interação com o processo produtivo, o layout da organização, 
dentre outros pontos que interferem diretamente na qualidade do produto final. Posteriormente foi elaborado e submetido um questionário a um dos sócios da organização de forma a possibilitar melhor compreensão do funcionamento da empresa. A partir das observações e dados qualitativos coletados foi elaborado em Diagrama de Causa e Efeito (Diagrama de Ishikawa) considerando-se como foco a redução de desperdícios. A partir de todos estes elementos foram elaboradas sugestões de atuação (CARVALHO et al., 2016).

Após a conclusão do projeto piloto, teve inicio o desenvolvimento simultâneo de vários estudos de caso realizados por diferentes grupos de alunos. O primeiro estudo de caso foi realizado em uma microempresa produtora de massas para vidro. Inicialmente foi convocada uma reunião com o gerente e todos os colaboradores da empresa na qual foram ouvidas todas as críticas em relação aos problemas enfrentados pela empresa. Utilizou-se nesta etapa a ferramenta Brainstorming.

O segundo estudo de caso foi desenvolvido em uma franquia do ramo alimentício (SUZART, AGUIAR, CARVALHO, 2017). A primeira fase do estudo foi constituída por uma abordagem qualitativa, na qual foram realizadas visitas técnicas, observações in loco e entrevistas informais com os gestores e colaboradores. Posteriormente foi aplicado um questionário.

Utilizou-se uma Curva ABC como forma de classificar os itens mais e menos vendidos a partir de uma análise de preços médios de mercado. Além disso, foram associados aos critérios de priorização os prazos de validade dos produtos. Posteriormente foi implementado o programa $5 \mathrm{~S}$ e metodologia FIFO (First in, first out) considerando o estoque um ambiente dividido em zonas para melhor direcionamento dos itens necessários Como uma última contribuição foi proposta a utilização de uma lista de verificação para o controle das bebidas consumidas pelos funcionários da organização.

\section{Pesquisa extensionista: resultados e discussões}

Conforme exposto anteriormente, o primeiro estudo de caso foi realizado em uma microempresa produtora de massas para vidro. Como resultado das observações iniciais elegeu-se o principal problema enfrentado pela empresa: o 
baixo índice de vendas. Constatou-se, inclusive, a possibilidade da não sobrevivência da empresa em médio prazo.

Após a coleta de dados foram elaborados vários Diagramas de Causa e Efeito (Diagrama de Ishikawa) e a partir das análises realizadas foi elaborado um plano de ação com o auxílio da ferramenta 5W2H (PACíFICO, ARAÚJO, CARVALHO, 2017).

O segundo estudo de caso foi desenvolvido em, uma franquia do ramo alimentício (SUZART, AGUIAR, CARVALHO, 2017), e identificou-se como maior desafio o controle de estoques.

Comparando-se os vários estudos de caso foi possível perceber a necessidade de integração de duas ou mais áreas da Engenharia de Produção de forma a resolver, ou no mínimo mitigar os diversos dos problemas encontrados.

O primeiro estudo de caso, utilizado como projeto piloto (fábrica de cortinas), trouxe como principal desafio a dificuldade de padronização de procedimentos, uma vez que o foco da empresa é oferecer soluções customizadas. Este fato possibilitou o despertar de vários questionamentos por parte dos alunos, o que deslocou o objetivo final do trabalho para o auxílio da redução de desperdícios, uma vez que a falta de padronização é inerente ao processo e pode ser até mesmo tratada como um diferencial competitivo.

O segundo estudo de caso (fábrica de massas de vidro) exigiu a integração de várias áreas como projeto de produto e marketing. Vale ressaltar que a crescente diminuição das vendas e a consequente ameaça a sobrevivência da organização está relacionada à mudança de mercado, que naturalmente passa a demandar produtos diferenciados. A primeira ideia foi o desenvolvimento de um novo produto capaz de impulsionar vendas mesmo que buscando um nicho de mercado diferente do nicho original (construção civil). Constada a impossibilidade desta opção, foi necessário elaborar um plano de ação apresentando claramente a necessidade de investimentos.

Foi recomendada ainda a elaboração formal do planejamento estratégico da empresa para melhor conhecimento dos seus concorrentes e posicionamento no mercado. Também foi sugerida a criação do plano de marketing para divulgação, pois constatou-se que clientes potenciais na região não têm conhecimento da empresa. Por fim, sugeriu-se a abertura das vendas 
para o mercado varejista no intuito de alcançar mais clientes e aumentar a receita da empresa (PACÍFICO, ARAÚJO, CARVALHO, 2017).

O terceiro e último estudo de caso possibilitou uma imersão no universo de empresas franqueadas. Geralmente a opção por uma franquia visa à oportunidade de diminuição das incertezas no momento da abertura de um novo negócio. Observou-se, entretanto, a rigidez quanto a procedimentos e um mix de produção excessivamente diversificado. A grande variedade de produtos impostos pela empresa franqueadora leva a desperdícios, considerando a comercialização de produtos perecíveis.

\section{Conclusões}

O objetivo final de qualquer empreendimento é a satisfação do cliente (seja ele interno ou externo). Entretanto, em muitos casos, não existe uma consciência a este respeito ou esta relação não é explícita sendo traduzida somente em termos de lucro, uma de suas consequências.

Espera-se que as microempresas participantes do projeto tenham resultados positivos quanto à gestão de seus recursos objetivando a melhoria do processo produtivo e aumento da competitividade. Para os alunos participantes espera-se que a oportunidade de conhecer o funcionamento e os problemas de uma empresa, e sugerir melhorias de acordo com seus conhecimentos teóricos, desenvolva uma visão holística e crítica, preparandoos melhor para o mercado de trabalho.

Com a finalização da atividade fica evidente que a utilização de uma estratégia gerencial, por intermédio da melhoria da qualidade, assegura uma melhor produtividade e por consequência a redução de desperdícios e dos custos. Propostas para essa solução, portanto, serão relatadas com dados qualitativos que comprovam que é possível gerar benefícios para a organização.

A interação dos alunos pesquisadores e a empresa caracteriza uma troca mútua de benefícios entre a Universidade e a comunidade de seu entorno. Conciliou-se o aprendizado teórico/prático para aplicação das ferramentas e busca das melhores propostas de soluções aos problemas 
apresentados pela empresa como contrapartida ao seu interesse em participar do processo.

Uma organização de qualquer natureza é composta por bens materiais e por seu potencial humano. Dentre estes dois itens, enquanto o primeiro é formado por componentes estáticos o segundo possui características intrinsecamente dinâmicas. O investimento no potencial humano, dentro das organizações, traz benefícios nem sempre tangíveis e mensuráveis em curto prazo, mas pode trazer um enorme crescimento refletido em aumento de produtividade em todos os sentidos.

\section{REFERÊNCIAS}

ANJOS, J.M F. et al. A contribuição do capital humano para o desenvolvimento: o caso da engenharia. In: PEREIRA, D.C.; ALBUQUERQUE, L. M.; CAMPOS, S. M. (Org.) Universidade e sociedade: desafios e perspectivas contemporâneos. Belo Horizonte: PUC Minas, 2015. Cap.26, p.197-201

ASSOCIAÇÃO BRASILEIRA DE ENGENHARIA DE PRODUÇÃO. Áreas e sub-áreas de Engenharia de Produção. Rio de Janeiro, 2008. Disponível em: $<\mathrm{http}$ ://www.abepro.org.br/interna.asp?p=399\&m=424\&s=1\&c=362> Acesso em: 27 de abril, 2016.

BATALHA, M. O. (Org.). Introdução à Engenharia de Produção. Rio de Janeiro: Elsevier, Campus, 2008.

CARPINETTI, L. C. R. Gestão da qualidade: conceitos e técnicas. 2. ed. São Paulo: Atlas, 2012.

CARVALHO, A. L.; NERY, M.S. Desenvolvimento de um Jogo Educacional Aplicável a Engenharia de Produção. In: ENCONTRO NACIONAL DE ENGENHARIA DE PRODUÇÃO, 35, 2015, Fortaleza. Anais... Rio de Janeiro: Associação Brasileira de Engenharia de Produção, 2015.

CARVALHO, A. L et al. Projeto de Extensão Piloto Aplicado a uma Micro Empresa de Produção Artesanal e Customizada. In: CONGRESSO BRASILEIRO DE EXTENSÃO UNIVERSITÁRIA, 7, 2016. Ouro Preto. Anais... [S.L.] Articulação Nacional de Extensão Popular (ANEPOP), 2016.

CASTRO, G. A. et al. A importância da Extensão na Formação Acadêmica dos Alunos do Curso de Engenharia Civil da PUC Minas Barreiro. In: SANABIO, D.; BARROS, E.B.R.(Org) Extensão PUC Minas: sujeitos, espaços e tempo de ação. Belo Horizonte: PUC Minas, 2017. 
CAUCHICK MIGUEL, P.A. Metodologia de Pesquisa em Engenharia de Produção e Gestão de Operações. 2. ed. Rio de Janeiro: Elsevier, 2012.

CRUZ, B.P.A. et al. Extensão Universitária e Responsabilidade Social: 20 anos de Experiência de uma Instituição de Ensino Superior. In: ENCONTRO DA ANPAD, 34,2010, Rio de Janeiro. Anais... Rio de Janeiro: Associação Nacional de Pós-Graduação e Pesquisa em Administração.

FEDERAÇÃO DAS INDÚSTRIAS DO ESTADO DE MINAS GERAIS . 2016. Disponível em: < http://www.fiemg.org.br > Acesso em: 10 de abril, 2016.

FÓRUM NACIONAL DE PRÓ-REITORES DE EXTENSÃO DAS UNIVERSIDADES PÚBLICAS BRASILEIRAS E SESU /MEC. Plano Nacional de Extensão Universitária. [S.L]: Edição Atualizada. Brasil, 2000/2001.

LAGE, G. F.; DIAS, V. C. Uma análise da Mostra Tecnológica do IPUC sob a ótica da Extensão Universitária. In: BRITTO, A.T. B. O.; GOMES, A.P.; SANABIO, D.; BARROS, E. B. R. (Org). Extensão PUC Minas: Conexão de conhecimentos, saberes e realidades. Belo Horizonte: PUC Minas, 2016. Resumo Expandido. p.259-362

LAROZINKI NETO, A.; LEITE, M. S. A abordagem sistêmica na Pesquisa em Engenharia de Produção. Produção, São Paulo, v.20, n. 1, p.1-14, jan./mar. 2010

MONTGOMERY, D.C. Introdução ao Controle Estatístico da Qualidade. 7. ed. Rio de Janeiro: LTC, 2016.

NEVES, P. O. et al. Plantio de espécies arbóreas nativas: uma alternativa para a melhoria nas condições socioambientais do município de São Gabriel-RS.

Revista Brasileira de Extensão Universitária, Chapecó, v. 8, n. 3, p. 149157, dez. 2017

PACÍFICO, C. C; ARAÚJO, L. L. S.; CARVALHO, A.L. Análise de um processo produtivo com demanda específica visando melhoria de desempenho financeiro. In: SANABIO, D.; BARROS, E. B. R. (Org.). Extensão PUC Minas: sujeitos, espaços e tempo de ação. Belo Horizonte: PUC Minas, 2017. Cap.15, p. 197- 207.

PALADINI, E. P. Avaliação estratégica da qualidade. 2. ed. São Paulo: Atlas, 2011.

PIOVEZAN, L. H.; BARBIN, F. J.; CARVALHO, M. M. C. Proposta de Método para a Formulação de Estratégia em Pequenas e Médias Empresas. Produção on Line, Florianópolis, v.8, n,2, jul 2008.

RODRIGUES, A. L. L et al. Contribuições da Extensão Universitária na Sociedade. Cadernos de Graduação - Ciências Humanas e Sociais . Aracaju, v. 1, n.16 , p. 141-148, mar. 2013 
RODRIGUES, M. V. Ações para a Qualidade. 5 ed. Rio de Janeiro: Elsevier, 2014.

SERVIÇO BRASILEIRO DE APOIO ÀS MICRO E PEQUENAS. Pequenos negócios promovem o desenvolvimento local. 2015. Disponível em: <http://www.sebrae.com.br/sites/PortalSebrae/artigos/Pequenosneg\%C3\%B3cios-promovem-o-desenvolvimento-local> Acesso em: 22 de jun, 2015.

SLACK, N.; CHAMBERS, S.; JOHNSTON, R. Operations management. 6. ed New York: Prentice Hall , 2010

SOUZA T.M, ATAYDE, H. M. Educação higiênico-sanitária dos feirantes do Mercadão 2000 e Feira do Pescado em Santarém-PA. Revista Brasileira de Extensão Universitária, Chapecó, v. 8, n. 3, p. 127-134, dez 2017.

SUZART, A. L. D.; AGUIAR, L. B.; CARVALHO, A. L.Análise organizacional de limitações e propostas de melhorias de uma empresa franqueada. In:

ENCONTRO MINEIRO DE ENGENHARIA DE PRODUÇÃO, 13, 2017, Juiz de Fora. Anais... Rio de Janeiro: Associação Brasileira de Engenharia de Produção, 2017.

TOLEDO, J. C. et al. Qualidade - Gestão e Métodos. Rio de Janeiro: LTC, 2013.

VENANZI, D.; SILVA, O. R. (Org.). Introdução à Engenharia de Produção.

Conceitos e Casos Práticos. Rio de Janeiro: LTC, 2016. 